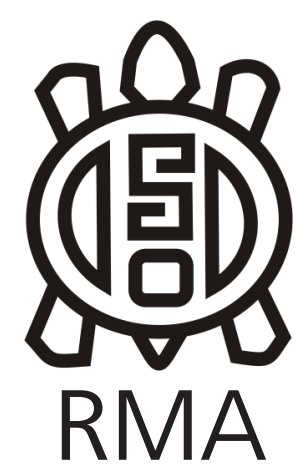

Antropología Social

\title{
La antropología oscura y sus otros. Teoría desde los ochenta
}

\author{
Sherry B. Ortner* \\ *Universidad de California, Los Ángeles \\ Traducción: Gustavo Blázquez** y María Cecilia Díaz*** \\ **Instituto de Humanidades-CONICET/Universdad Nacional de Córdoba; \\ Departamento de Antropología, (FFyH-UNC) y CIFFyH-UNC. \\ E-mail: gustavoblazquez3@hotmail.com \\ ***Departamento de Antropología, (FFyH-UNC) y CIFFyH-UNC. \\ E-mail: mcecilia.diaz@gmail.com
}

N. de T.: Ortner, Sherry B. 2016. "Dark Anthropology and its others: Theory since the eighties". In Hau: Journal of Ethnographic Theory 6(1): 47-73. Agradecemos especialmente a la autora y a la revista por la posibilidad de traducir y publicar este artículo. También quisiéramos agradecer a Ivana Wolff, Rodolfo Fenoglio e Ignacio Muñiz por las revisiones y correcciones. El texto original tiene libre acceso en: https://www.haujournal.org/index.php/hau/article/view/hau6.1.004.

\begin{abstract}
In this article I consider several emergent trends in anthropology since the 1980s against a backdrop of the rise of neoliberalism as both an economic and a governmental formation. I consider first the turn to what I call "dark anthropology," that is, anthropology that focuses on the harsh dimensions of social life (power, domination, inequality, and oppression), as well as on the subjective experience of these dimensions in the form of depression and hopelessness. I then consider a range of work that is explicitly or implicitly a reaction to this dark turn, under the rubric of "anthropologies of the good," including studies of "the good life" and "happiness," as well as studies of morality and ethics. Finally, I consider what may be thought of as a different kind of anthropology of the good, namely new directions in the anthropology of critique, resistance, and activism.
\end{abstract}

Keywords: anthropological theory; neoliberalism; Marx; Foucault; well-being; morality; critique; resistance

\section{Resumen}

En este artículo considero varias tendencias emergentes en la antropología desde los 80, en el contexto de ascenso del neoliberalismo en tanto formación económica y gubernamental. Considero primero el giro hacia lo que llamo "antropología oscura", es decir, la antropología que se enfoca en las dimensiones difíciles de la vida social (poder, dominación, desigualdad y opresión), como también en la experiencia subjetiva de esas dimensiones, en forma de depresión y desesperanza. Considero luego un abanico de trabajos que se presentan como una reacción explícita o implícita a este giro oscuro bajo la rúbrica de "antropologías de lo bueno" ${ }^{1}$, incluyendo estudios de "la buena vida" y la "felicidad", como también estudios de moralidad y ética. Finalmente, considero lo que podría ser pensado como un tipo diferente de antropología de lo bueno, es decir, nuevas direcciones en la antropología de la crítica, la resistencia y el activismo.

Palabras clave: teoría antropológica; neoliberalismo; Marx; Foucault; bienestar; moralidad; crítica; resistencia

1[N. de T.] Aquí optamos por esta formulación para la traducción de "anthropologies of the good", considerando que el universo semántico de "good" remite a "bienestar", "placentero", "alta calidad", "exitoso", "amable", "correcto" y "saludable". De ese modo, creemos que permite contemplar las tendencias analíticas que la autora identifica y reúne en tal expresión.

El trabajo académico, al menos en las ciencias sociales, no puede separarse de las condiciones del mundo real en el que se desarrolla. Los marcos teóricos que usamos y los fenómenos que elegimos explorar son afectados de mil maneras por las circunstancias políticas, económicas y culturales en las cuales llevamos a cabo nuestra investigación, aun cuando tal investigación sea sobre el pasado distante o sobre lugares alejados. Como los académicos en ciencias sociales han discutido por décadas, incluso el estudio de objetos físicos y fuerzas remotas a los asuntos humanos está condicionado por las circunstancias históricas que rodean la investigación.

El caso analizado en este artículo es la transformación de la antropología contemporánea en relación, entre otras cosas, con el comienzo del orden socio-económico- 
político llamado "neoliberalismo". Si bien definiré específicamente el término más adelante, necesito decir algunas palabras al respecto aquí. En el período bajo discusión -aproximadamente desde mediados de 1980 hasta mediados de 2010- el neoliberalismo como una forma nueva y más brutal del capitalismo se expandía rápidamente sobre el planeta. En el frente interno, la economía norteamericana en los 80s comenzó lo que el historiador Robert Brenner (2006) ha llamado "el largo declive", que culminó en el casi colapso de la bolsa de valores en 2008, seguido por una recesión profunda. Los bancos, que se habían vuelto "demasiado grandes para caer", fueron rescatados luego de la crisis con el dinero de los contribuyentes y rápidamente recompensaron a sus ejecutivos de alto nivel con bonos gigantescos. Sumado a esto, la brecha entre ricos y pobres en Estados Unidos creció sostenidamente durante este periodo, eventualmente superando la brecha existente antes de la Gran Depresión de la década de 1930 -y los economistas se vuelven más pesimistas acerca de la reversión de esta tendencia (Piketty, 2014). Mientras tanto, en el frente internacional, el Fondo Monetario Internacional (FMI) y el Banco Mundial impulsaron políticas económicas neoliberales que esencialmente aplastaron las economías de algunas de las naciones más pequeñas y pobres del mundo (Ferguson 1999; Duménil and Lévy 2004; Harvey 2005; Klein 2007; Ortner 2011).

Admito que escribo desde la perspectiva de los Estados Unidos, donde la situación ha sido extrema, particularmente con respecto a la transferencia ascendente de riquezas y su impacto en las políticas norteamericanas, y en relación al crecimiento de una profunda desigualdad. Por supuesto, hay un gran margen de variación entre casos locales y nacionales. Aihwa Ong nos advierte que no debemos ver al neoliberalismo "como una marea... que se extiende desde los países dominantes a los más pequeños" (2006:12) y, en lugar de eso, nos urge a mirar los "ensamblajes" complejos (Ong y Collier 2005) en los cuales el neoliberalismo entra en diferentes tiempos y lugares. Además de este tipo de variaciones, la situación está evolucionando activamente en diferentes partes del mundo, incluso mientras escribo. Los antropólogos están empezando a documentar adaptaciones creativas al neoliberalismo, como también movimientos de resistencia contra él-y, en cualquier caso, a algunos países claramente les está yendo mejor que a otros. Así que, una vez más, las discusiones en este artículo no asumen un desarrollo uniforme del neoliberalismo en todas partes, sino que están escritas en el contexto del caso estadounidense, y en un período en el que las condiciones fueron particularmente "oscuras".

Este artículo no intenta sugerir que el neoliberalismo explica todas las cosas malas que suceden en los Estados Unidos y globalmente. Las cuestiones de raza y género, y de violencia religiosa y étnica tienen sus propias historias locales y sus propias dinámicas internas, aunque no escapan de sus vínculos con las formas neoliberales de economía y gobernanza donde estas aparecen.

Y finalmente, este artículo no pretende cubrir todos los desarrollos en antropología en el periodo bajo discusión. Muchos desarrollos interesantes e importantes no son discutidos, incluyendo el "giro ontológico" (p. ej., Costa y Fausto 2010; Graeber 2015; Salmond 2014; Tsing 2015), el "giro afectivo" (p. ej., Mankekar 2015; Mazzarella 2009; Rutherford 2016), y el giro hacia la "teoría etnográfica" (da Col y Graeber 2011), entre otros. En lugar de ello, este ensayo se enfoca en un grupo de tres áreas interrelacionadas de trabajo que visualizo como vinculadas a los funcionamientos problemáticos del neoliberalismo: (1) la emergencia de lo que llamo "antropología oscura", incluyendo tanto la teoría como la etnografía; (2) la emergencia dialécticamente vinculada de lo que he llamado "antropologías 'de lo bueno'"; y (3) la re-emergencia del estudio de la "resistencia", que tomo como un término paraguas para un conjunto de nuevos trabajos críticos, etnográficos y teóricos.

\section{El triunfo de la antropología oscura}

Como ya discutí en un ensayo anterior (Ortner 1984), en los 60 y 70 la antropología norteamericana estuvo dominada por una división entre un ala "culturalista" liderada por Clifford Geertz (p. ej., 1973) y sus estudiantes, y un ala marxista o materialista, liderada por Eric Wolf ( $p$. ej., 1982) y sus colegas (p. ej., Hymes 1972). Inspirados principalmente por Max Weber, Geertz y sus seguidores estaban interesados en nuevas maneras de pensar sobre la cultura -sobre cómo la cultura provee significado a la vida de las personas, y cómo los antropólogos pueden llegar a entender esos significados. Wolf y compañía, por otro lado, estaban inspirados principalmente por Marx, y estaban interesados en las maneras en las cuales las vidas de las personas están menos moldeadas por su cultura y más por las fuerzas económicas y políticas en juego, tanto local como globalmente. Desde el punto de vista de los culturalistas, el trabajo de los académicos en economía política era reduccionista: los motivos de las personas eran reducidos a "intereses" simplistas, y sus vidas eran vistas como reflejos de fuerzas mecánicas. Desde el punto de vista de los materialistas, por otro lado, el trabajo de los culturalistas era básicamente insuficiente: al tratar la cultura como los textos literarios, ignoraban las adversas realidades de poder que impulsaron gran parte de la historia humana.

La perspectiva culturalista prevaleció durante las décadas de 1960 y 1970, al menos en Estados Unidos. Al mismo tiempo, y en parte superponiéndose al enfoque de la economía política marxista, tomaban forma nuevas críticas que también insistían en la importancia de tener en cuenta cuestiones de poder, desigualdad, dominación y explotación. Tuvo gran importancia la emergencia de la teoría poscolonial, que atravesó un amplio rango de disciplinas. En antropología, una publicación temprana y muy importante fue la colección Anthropology and the 
colonial encounter (1973) ${ }^{1}$ de Talal Asad. Los comienzos de 1970 también vieron el crecimiento de los estudios feministas, nuevamente en un amplio rango de disciplinas. Las entradas principales en este campo en antropología fueron Woman, culture and society (1974) de Michelle Rosaldo y Louise Lamphere, y Toward an anthropology of women (1975) de Rayna (Rapp) Reiter. Aunque la raza no pasó a primer plano de la misma manera hasta un tiempo después (sin embargo, véase Szwed 1972), los estudios críticos del colonialismo y el poscolonialismo contenían una fuerte dimensión de crítica racial.

Los practicantes de estos nuevos tipos de trabajo no necesariamente estaban de acuerdo entre sí: la gente de la economía política tendía a ignorar el género (como también la raza); los académicos colonialistas a menudo tenían problemas con la economía política; y los académicos de género no acordaban necesariamente entre sí. Pero todos estaban de acuerdo, al menos implícitamente, en que la antropología tenía que comenzar a prestarle atención a los temas del poder y la desigualdad, y a la larga, empezando alrededor de 1980, estos comenzaron a prevalecer. Las cuestiones de poder y desigualdad han llegado a dominar el paisaje teórico, tanto al nivel de los "ancestros" teóricos (Marx, Weber, etc.), como al nivel de los temas más prominentes de la investigación contemporánea (colonialismo, neoliberalismo, patriarcado, desigualdad racial, etc.). Llamo a esto el ascenso de la "antropología oscura", esto es, una antropología que enfatiza las dimensiones duras y brutales de la experiencia humana, y las condiciones estructurales e históricas que las producen. Este giro hacia la antropología oscura (incluyendo tanto "teoría oscura" y "etnografía oscura") es, al menos en parte, una respuesta a las críticas internas subrayadas previamente, pero también una respuesta -como sostengo- a las condiciones crecientemente problemáticas del mundo real bajo el neoliberalismo. ${ }^{2}$

Miremos primero el cambio en los ancestros teóricos. En 1971, Anthony Giddens publicó uno de los libros de ciencia social más vendidos de todos los tiempos, El capitalismo y la moderna teoría social Giddens sostenía que la teoría social moderna fue inaugurada por el trabajo de Karl Marx, y por los de Émile Durkheim y Max Weber, respondiendo en parte a la obra de Marx. Esto representó una ruptura significativa con convenciones previas en las ciencias sociales, las cuales, por diversas razones a ambos lados del Atlántico, habían dejado en su mayor parte la obra de Marx por fuera del canon de la teoría. Probablemente

\footnotetext{
${ }^{1}$ Cinco años después Edward Said publicó Orientalismo (1978), que fue aclamado como una obra de gran originalidad, aunque sus puntos de vista eran prácticamente idénticos a los de Asad. ${ }^{2}$ Obviamente existen muchos otros factores detrás de este cambio en ese momento. Entre otras cosas, se podrían observar más de cerca las otras tensiones dentro del campo, particularmente aquellas que rodean la publicación de Writing Culture (Clifford y Marcus 1986). Estoy en deuda con Johnny Parry por destacar este punto, pero desafortunadamente no puede ser tratado en los límites de este artículo.
}

el conjunto Marx-Durkheim-Weber todavía es enseñado como fundacional en muchos -si no en la mayoría- de los cursos principales de grado, pero yo propondría que el rol de estas tres figuras en la definición de los temas teóricos principales en el campo se ha modificado considerablemente, como parte del cambio de foco que se discute aquí. En la medida en que Durkheim estaba asociado principalmente con una perspectiva funcionalista estática, y en tanto mostró relativamente poco interés en el poder y la desigualdad, su influencia se ha desvanecido en gran medida, aunque están surgiendo ciertas tendencias neo-durkheimianas a las que volveré más adelante. En la medida en que el trabajo de Weber ha sido asociado principalmente con asuntos de cultura, y de hecho con el concepto en gran parte apolítico de cultura de Clifford Geertz, la influencia de Weber también ha disminuido, aunque su trabajo sobre los orígenes culturales del capitalismo y las formas de dominación lo mantienen más activamente en escena. Al mismo tiempo, la influencia de Marx ha crecido enormemente, en muchos aspectos. Su modelo general de la modernidad capitalista, acentuando la explotación económica y la desigualdad de clases es, si no hegemónico, entonces ampliamente aceptado, aunque ha sido desmenuzado y complejizado en miles de formas por los neo- y post- marxismos. Asimismo, la versión dominante del concepto de cultura usado en la actualidad es el concepto de inspiración marxista de hegemonía, un refinamiento político del concepto antropológico de cultura a partir del concepto marxista de ideología (R. Williams 1977). Al mismo tiempo, la influencia de Foucault, quien fue casi invisible para la antropología de habla inglesa en 1970, se ha expandido en proporciones importantes. Foucault desarrolló un marco teórico que está profundamente preocupado con formas y modalidades del poder. Él nos ha dado todo un nuevo vocabulario del lenguaje del poder, incluyendo "gubernamentalidad", "biopolítica", "subjetivación" y más, los cuales buscan captar las múltiples maneras en las que el poder se despliega, grosera y sutilmente, en el mundo moderno (Foucault 1977, 1980, 2008; véase también Burchell, Gordon y Miller 1991).

Las obras de Marx y Foucault, cada una a su manera, definen y representan el cambio hacia la "teoría oscura", teoría que nos pide ver el mundo casi enteramente en términos de poder, explotación y desigualdad crónica generalizada. Parte del trabajo de Foucault es casi un ejemplo perfecto de este concepto, una teoría virtualmente totalizante del mundo en la cual el poder se encuentra en cada resquicio de la vida, y en la cual no hay un afuera del poder (p. ej., Historia de la sexualidad, 1980). Por supuesto, su pensamiento evolucionó a lo largo de su carrera y algunos de sus últimos trabajos se alejaron de la problemática implacable del poder (especialmente Tecnologías del yo [Martin, Gutman y Hutton, 1988]). Sin embargo, pienso que es justo decir que es el Foucault oscuro -el Foucault del panóptico, de Vigilar y castigar (1977), del poder capilar y las múltiples formas de 
gubernamentalidad- el que ha tenido la mayor influencia en la teoría antropológica sociocultural. Lo mismo puede sostenerse respecto a Marx. Aunque hay ciertos aspectos optimistas de la teoría marxista, el Marx en juego en la teoría antropológica actualmente es sobre todo el Marx oscuro, que enfatiza el enriquecimiento de los ricos y poderosos a expensas de los pobres y sin poder, y la implacable expansión global del capitalismo como una formación social y económica brutal y deshumanizante.

Si el conjunto de ancestros teóricos ha variado hacia la "teoría oscura", también muchos de los sujetos y objetos de la investigación etnográfica han cambiado hacia una materia oscura. El principal ejemplo, que será discutido en este artículo, es el giro generalizado hacia el estudio del neoliberalismo y sus efectos. Pero antes de llegar a eso, es importante observar brevemente, en la misma línea, la explosión del estudio de los asuntos coloniales.

Es difícil exagerar el grado en el cual el marco colonial ha transformado la manera en que la antropología se vincula con el mundo en la actualidad. En mis estudios de grado en antropología y en mi formación durante el posgrado, la palabra "colonialismo" era, en lo que puedo recordar, difícilmente mencionada. Los antropólogos interesados en el colonialismo en esos tiempos, como Bernard Cohn (1996), eran considerados figuras raras y marginales. Pero a partir del trabajo de Talal Asad, Edward Said y eventualmente muchos otros, el campo como un todo se transformó radicalmente. Se volvió imposible mirar al Ilamado Tercer Mundo sin entenderlo como parte de una historia de expansión colonial (y para los marxistas, capitalista). La división del mundo en naciones ricas y pobres que tenemos hoy se volvió inteligible como resultado de, entre otras cosas, la extracción de riqueza de las colonias en el pasado. Muchas formaciones sociales y culturales que aparecen en trabajos antropológicos tempranos como "eternas" empezaron a aparecer de manera diferente contra el telón de fondo de la historia colonial (véase, p. ej., Mamdani 1996 sobre el conflicto ético; Dirks 2001 sobre la casta). Las historias detalladas del encuentro colonial (Comaroff y Comaroff 1991, 1997 en Africa; Merry 2000 en Hawaii) cuentan relatos de la reconstrucción implacable de pueblos y culturas bajo las condiciones de la penetración y dominación occidental (i.e., misionera y colonial), que simplemente no estaban en la agenda cuando yo era estudiante de antropología y que representan una de las tantas transformaciones oscuras del campo bajo discusión.

En última instancia, los marcos poscolonial y neoliberal comenzarán a converger, ya que la mayoría de las postcolonias serán neoliberalizadas por uno u otro mecanismo. Aquí, entonces, me referiré al fenómeno del neoliberalismo.

\section{Neoliberalismo en el mundo y en la antropología}

Si el neoliberalismo (entre otras cosas) se encuentra detrás del ascenso de la teoría oscura, entonces podemos decir que también está a su frente: el neoliberalismo y sus efectos se han vuelto objetos de estudio y marcos para entender otros objetos de estudio en un amplio espectro de trabajo antropológico (para comenzar, véase Greenhouse 2010; Gusterson y Besteman 2010). Como con el colonialismo, es difícil sobreestimar el grado en que estas cuestiones han llegado a dominar el campo. Me centraré aquí en dos de los mayores grupos de trabajo que se han acumulado sobre este tema desde 1980. Uno de ellos comienza con el neoliberalismo como un tipo específico de sistema económico y traza el impacto de las políticas económicas neoliberales tanto en el Norte como en el Sur Global. El otro comienza con el neoliberalismo como una forma específica de gubernamentalidad y rastrea la variedad de formas que adopta en diferentes contextos. Examinaré estas dos áreas separadamente, pero destaco aquí que no se trata de una distinción clara y rápida, y que hay mucha superposición entre los dos tipos de trabajo. Destaco también que éste no pretende ser un análisis exhaustivo del neoliberalismo como tal, sino que es un intento de distinguir algunas de las líneas de trabajo más prominentes que los antropólogos han desarrollado al respecto.

\section{Economía: la acumulación por la desposesión}

David Harvey comienza su breve e indispensable historia del neoliberalismo con la siguiente definición básica:

“El neoliberalismo es, en primer lugar, una teoría de prácticas político-económicas que afirma que la mejor manera de promover el bienestar del ser humano es favorecer el libre desarrollo de las capacidades y libertades empresariales del individuo en un marco institucional caracterizado por derechos de propiedad privada fuertes, mercados libres y libertad de comercio. El rol del Estado es crear y preservar el marco institucional apropiado para tales prácticas" (2005:2)

Este marco institucional incluye, entre otras cosas, la remoción de las regulaciones del gobierno sobre los negocios, la reducción del poder de los trabajadores para hacer demandas, el recorte de la fuerza de trabajo en sí misma, la privatización de los bienes públicos y las instituciones; y la reducción radical de los programas de asistencia social para los pobres. El efecto de todo esto ha sido el crecimiento de la desigualdad extrema dentro y entre las naciones, con un puñado de individuos ricos volviéndose dramáticamente ricos, las masas de pobres volviéndose significativamente más pobres, y la clase media resistiendo -donde lo logra- a fuerza de un trabajo extremadamente duro y de auto explotación. Para Harvey se trata de un sistema de "acumulación por desposesión" (ibíd.:159), en el que la riqueza es redistribuida hacia arriba, dentro y entre las economías capitalistas.

Es importante notar que el neoliberalismo emergió en el contexto de un largo período de prosperidad en el Norte Global después de la Segunda Guerra Mundial, produciendo una sensación de seguridad en esa época y un 
gran optimismo hacia el futuro. En los Estados Unidos esto tomó la forma de la idea del "sueño americano", la idea de que cualquiera podía alcanzar seguridad económica para sí mismo y su familia si simplemente trabajaba lo suficiente y tenía las actitudes correctas (véase, p. ej., Ortner 2006). Globalmente, tomó la forma de programas de "modernización" y "desarrollo", con la idea de que las naciones pobres del Sur Global podían "ponerse al día" con una inyección razonable de recursos materiales y tecnológicos de las naciones "más desarrolladas". Aunque a un nivel todo esto era ideológico, también se fundaba en condiciones materiales reales: en el Norte Global, las economías estaban en expansión y los niveles de inequidad estaban cayendo; en el Sur Global, muchas naciones pobres experimentaron un periodo de crecimiento económico y de confianza en el futuro.

Comenzando 1980, con Reagan en los Estados Unidos y Thatcher en el Reino Unido, todo esto empezó a cambiar. Bajo las políticas neoliberales de recortes y deslocalización [offshoring] para aumentar las ganancias, muchas fábricas y otras unidades de producción fueron cerradas, y cientos de miles de personas perdieron sus empleos. Al mismo tiempo, bajo las teorías neoliberales acerca de la importancia de achicar el estado y abolir, o al menos reducir al máximo, prácticamente todos los programas de ayuda social, muy pocos recursos estuvieron disponibles para las personas y familias desempleadas o subempleadas. Incluso estas teorías fueron impuestas a otras naciones como el precio de aceptar préstamos de las potencias mundiales. Muchos individuos y naciones enteras (nuevamente, con muchas variaciones) comenzaron a perder rápidamente los recursos que habían acumulado en el pasado.

Desde los tempranos años 90, los antropólogos empezaron a narrar las maneras en las que ese proceso ocurría, tanto en casa como afuera, incluyendo no solo los efectos económicos negativos, sino también la emergencia de nuevas "estructuras de sentimiento" (R. Williams, 1977) dolorosas. En 1993, Pierre Bourdieu y colegas publicaron un largo volumen titulado The weight of the world: Social suffering in contemporary society. Basado en entrevistas con personas de clase trabajadora nacidas en Francia e inmigrantes, el trabajo presenta testimonios en primera persona y reflexiones académicas acerca de las miserias de la vida en la nueva economía. Tomo un ejemplo de las más de seiscientas páginas de entrevistas en su mayoría inquietantes. Bourdieu cita a un sindicalista sobre las consecuencias del desempleo: "Hay mucho dolor, mucho sufrimiento mental y físico, la gente sufre y sufre... el desempleo nos divide y saca lo peor de nosotros, individualismo, celos, envidia..." (Bourdieu et al. 1993:318). En 1999, James Ferguson publicó un relato de cómo Zambia, que parecía ser uno de los lugares más prometedores para la modernización exitosa y el desarrollo en África, ha sido tanto desindustrializada como neoliberalizada, y cómo existe una sensación generalizada de desvanecimiento del anterior ánimo de promesa. Él describe la sensación entre muchos zambianos contemporáneos de lo que llama "abyección" o "ser expulsado [y] desechado" (1999:236), y de desconexión, de estar "desconectado" del sistema mundial, un lugar donde -entre otras cosas- las mayores aerolíneas no paran más. En 2013, Anne Allison publicó un extenso estudio de lo que ella llama "Japón precario", describiendo las numerosas maneras en las cuales los japoneses de todas las edades sufren en las nuevas condiciones de inseguridad económica y social. En el capítulo de lo que ella denomina "refugismo ordinario" ["ordinary refugeeism"], discute el incremento de las tazas de suicidio y de las formas de aislamiento social, incluyendo la renuncia voluntaria a tener una vivienda de los "refugiados de los net cafés" y el ahora bien conocido fenómeno de los hikikomori, o jóvenes que se aíslan en sus cuartos, en casa de sus padres, y se rehúsan a salir por años.

Volviendo a Estados Unidos, los antropólogos comenzaron por observar las consecuencias de la desindustrialización de la economía en 1980. Cuando las fábricas y otras instalaciones industriales (por ejemplo, las minas) cerraron, miles de trabajadores fueron expulsados del trabajo, y la clase obrera estadounidense básicamente se derrumbó, económica y políticamente. Kathryn Dudley (1994) realizó un estudio del extendido impacto del cierre de la planta automotriz Chrysler en la ciudad de Kenosha, Wisconsin; el subtítulo de su libro es un juego entre el cierre de la planta automotriz y la pérdida de esperanza: "el fin de la línea". Y Christina Walley (2013) escribió una etnografía personal sobre el cierre de Wisconsin Steel Works en el sudeste de Chicago y el impacto sobre su familia, como también sobre el resto de la comunidad. Ella nos habla, entre otras cosas, sobre la depresión creciente de su padre por el desempleo, y su rechazo eventual a afeitarse, cambiar sus ropas o salir de la casa (ibíd.: 128). ${ }^{3}$

El cierre de fábricas en 1980 fue representado como el resultado necesario de la globalización, en la que los avances de las tecnologías de comunicación y transporte proveían acceso a mercados de trabajo más baratos. ${ }^{4}$ La industria norteamericana se representaba a sí misma como "teniendo que" derivar a terceros la producción para poder competir en el mercado global. Incluso, hay un modo en el que el neoliberalismo parece fomentar un tipo de actitud desdeñosa hacia las clases trabajadoras y los pobres, más allá de la necesidad de la ganancia. Esta actitud está encarnada en el achicamiento del estado y en la muerte lenta de todas sus funciones de servicio social descriptas previamente, y actúa de muchas maneras

${ }^{3}$ De manera similar, el documental Roger and me (1989) de Michael Moore registra la devastación de su ciudad natal, Flint, Michigan, como resultado del cierre de la planta automotriz General Motors que se encontraba allí.

"Esos "mercados de trabajo más barato" también han sido estudiados por antropólogos, comenzando con Ong (1987) e incluyendo estudios de las condiciones de trabajadores a lo largo de la frontera entre México y Estados Unidos (FernándezKelly 1983; Sklair 1993; Wright 2001). 
diferentes en distintas partes de la sociedad. Así, más allá de la desindustrialización, vemos un tipo de guerra más activa contra los pobres, de nuevo documentada etnográficamente en numerosos lugares y contextos. Por ejemplo, Jane Collins (2010) ha observado las maneras en las cuales Wal-Mart destruye comunidades enteras y empobrece a sus trabajadores en casa y en el extranjero. Ella sostiene que esta destrucción no es un subproducto accidental de un plan de negocios racional, sino que "hay una relación integral, intencional y multifacética entre Wal-Mart y la pobreza" (ibíd.:99). Brett Williams ha documentado el crecimiento de una industria de préstamos de dinero altamente rentable que opera sobre todo en vecindarios pobres, y de "una industria de cobro de deudas agresiva y abusiva" que persigue a los deudores cuando no pueden pagar sus deudas. Ella concluye: "con mal crédito ya no eres más un humano" (Williams 2010:230; véase también Wacquant 2009 sobre "castigar al pobre").

Y para un estudio etnográfico en profundidad sobre el funcionamiento del neoliberalismo en una comunidad pobre devastada por el desastre, podemos ir al análisis de Vicanne Adams sobre el antes y el después del Huracán Katrina en Nueva Orleans en 2005. La inundación que destruyó muchos vecindarios pobres fue causada no por el huracán en sí, sino por el rompimiento de los diques que habían protegido a la ciudad de las aguas que la rodeaban. El trasfondo del desastre, entonces, es la negligencia y el deterioro de los diques, y Adams nos habla de la creciente influencia en el gobierno federal de grandes contratistas privados como Halliburton y Blackwater, quienes jugaron un rol mayor en desviar fondos y atención hacia áreas de trabajo más rentables, dejando los diques altamente vulnerables. Por otro lado, luego del huracán y de la inundación masiva se declaró una emergencia nacional, pero la Federal Emergency Management Authority (FEMA) estaba también en connivencia con estos y otros grandes contratistas, quienes obtuvieron los contratos para ocuparse de la ayuda de emergencia aunque no tenían experiencia en tales situaciones. Adams llama a la recuperación un "desastre de segundo orden" (2013:1), en el cual casi nadie recibió la ayuda que le había sido prometida, y los barrios y vidas quedaron en ruinas.

En suma, en los peores casos, es decir entre los pobres, hay un incremento de la pobreza, un deterioro de las condiciones de vida, y una depresión y desesperanza crecientes. Pero incluso en las clases medias hay una sensación de declive de la buena suerte (Newman, 1993), pérdida de optimismo y gran inseguridad sobre el presente y el futuro.

Estos ánimos sombríos no dejaron de ser registrados en la cultura pública -cine, televisión, publicidad, medios impresos-, que debe incluirse en cualquier definición amplia de etnografía. Vale la pena notar, entonces, que el concepto de Anne Allison's "ordinary refugeeism", mencionado previamente, estaba de hecho inspirado en un documental llamado (en traducción) Net café
refugees-The drifting poor (Mizushima 2007) que tuvo un gran impacto en Japón cuando se estrenó. Mi libro reciente, Not Hollywood (Ortner, 2013a) narra el ascenso de las películas independientes norteamericanas en 1980 y 1990. Los cineastas independientes se enorgullecen de estar comprometidos con un realismo duro contra lo que ellos ven como el brillo falso de las películas de Hollywood. En muchas, incluso la mayoría de las películas independientes, la violencia emocional es la forma prevalente de las relaciones sociales, la oscuridad y la depresión son el ánimo dominante y los finales felices son virtualmente inexistentes. Finalmente, Cruel Optimism de Lauren Bernant, también basado en lecturas de la cultura pública, provee una reflexión brillante sobre las "fantasías desgastadas" de la "movilidad ascendente, seguridad laboral, igualdad política y social e intimidad vívida y durable" en la era neoliberal, y las consecuencias afectivas de estos aspectos deteriorados de la buena vida (2011:3). El tropo central del libro es la frase que le da título, "optimismo cruel", definido como una relación "cuando algo que deseas es en realidad un obstáculo para tu florecimiento" (ibíd.:1) y se refiere al hecho de que las personas todavía se aferran a los accesorios del viejo orden, aunque ya no sean funcionales en el mundo que habitamos ahora.

Es importante mencionar aquí que la mayor parte de esos trabajos no termina con un tono completamente negativo. Muchos de ellos proveen indicaciones sobre formas locales de crítica y de resistencia al nuevo orden. Por ejemplo, en Precarious Japan, Allison nos presenta a Yuasa Makoto, cofundador de Reverse Poverty Network y codirector de Moyai, un centro de atención para las personas sin hogar que necesitan ayuda social y asistencia práctica para reencausar sus vidas. Y en Not Hollywood, dedico un capítulo a la filmación políticamente activa de documentalistas que nos muestran no solo los lados oscuros del neoliberalismo discutidos aquí, sino también a individuos y grupos que intentan cambiar estas tendencias. Discutiré sobre resistencia y activismo más profundamente en la parte final de este artículo.

\section{Gubernamentalidad: la república del miedo 5}

El concepto de gubernamentalidad de Foucault ha sido altamente productivo y ha inaugurado diferentes líneas de trabajo en antropología. Una línea sigue sus argumentos acerca de la producción del sujeto liberal -el individuo capaz de escoger libremente- como la forma hegemónica de gubernamentalidad en el mundo neoliberal (Rose 1996; Brown 2003). En ese contexto, los antropólogos se han preguntado no tanto sobre el impacto del neoliberalismo como política económica, tal como fue discutido en la sección anterior, sino sobre las maneras en las cuales la sociedad está siendo neoliberalizada, esto es, hecha para operar según principios neoliberales de eficiencia de mercado, y ensamblada para la producción de sujetos neoliberales emprendedores y auto-administrados. Otra

\footnotetext{
${ }^{5}$ La frase es del título de Lancaster (2010).
} 
línea de pesquisa, que también se deriva directamente de Foucault, ha seguido los vínculos entre gubernamentalidad y la regulación de poblaciones. Así, Aihwa Ong explora las formas de gubernamentalidad realizadas por estados y otros agentes al regular la movilidad voluntaria o involuntaria de "sujetos móviles", ya sean elites como los hombres de negocios chinos o los "esclavos virtuales" como los trabajadores domésticos del sur de Asia (1999, 2006; véase también Gregory 2007). Y Akhil Gupta (2012) ha examinado la "violencia estructural" ejercida contra los pobres a través de los mecanismos de gobierno de la burocracia en la India.

Sin embargo, hay otra línea de indagación antropológica sobre gubernamentalidad ampliamente definida, que ha recibido de alguna manera menos atención: lo que Roger Lancaster llamó "gobernanza punitiva" (2010:74) o la proliferación de formas de violencia del estado contra sus ciudadanos. La relación entre el neoliberalismo y la prolífica violencia estatalmente organizada, que incluye -pero no se limita a- la guerra, ha sido explorada en profundidad por Naomi Klein (2007). Su argumento central en The shock doctrine es que los incidentes de extrema violencia, sean desastres naturales o golpes políticos, han conformado en todas partes la estrategia para imponer medidas económicas neoliberales en las políticas, en municipios como Nueva Orleans y en países enteros como Chile o Polonia. Ella también observa una extendida conexión entre la imposición de medidas económicas neoliberales y el uso de la tortura, en Estados Unidos y en otras partes. Cita al escritor uruguayo Eduardo Galeano, quien dice "¿Cómo salvar esa desigualdad si no es a golpes de picana eléctrica?" (ibíd.:7).

En los Estados Unidos, este tipo de violencia del estado contra su propia ciudadanía es particularmente visible, entre otras cosas, en la proliferación de prisiones. Roger Lancaster ha descripto los Estados Unidos como, en la frase de Foucault, un "estado carcelario", argumentando que "la gobernanza punitiva es la lógica cultural real del neoliberalismo" (2010:63, 74). Lancaster cita estadísticas a los efectos de que "los Estados Unidos se encuentran primeros en el ranking de taza de encarcelamiento (uno de cada 99 adultos residentes está tras las rejas) y en el número absoluto de personas encarceladas (2.26 millones)" (ibíd.:64). Además, una alta proporción de esos prisioneros son afroamericanos que se ven doblemente afectados por la pobreza y la raza. Pero un estado carcelario no es simplemente uno que tiene muchos presos; es uno en el cual "un sistema de prisión inflado suple las normas de la gobernanza en general" (ibíd.:63). Este, entonces, es el otro lado de la gubernamentalidad neoliberal: si en el esquema clásico foucaultiano la gubernamentalidad neoliberal funciona a través del consentimiento de sujetos ostensiblemente libres, una de las formas en las que proclama la seguridad de esa libertad es a través del encarcelamiento $y$, de hecho, en muchos contextos, matando a aquellos que supuestamente la amenazan -no solo criminales, por supuesto, sino también a aquellos construidos como "terroristas" en el mundo post 9/11.

Si, como Lancaster sostiene, el estado carcelario "suple las normas de la gobernanza en general" también proporciona un tipo de modelo inverso y perverso de la vida social cotidiana. En su historia social de Los Ángeles, el crítico social Mike Davis (1992) presenta un capítulo llamado Fortress $L . A$, en el que explora la nueva arquitectura de Los Ángeles y las maneras en las que una cierta estética de la prisión ha tomado la ciudad. Muestra, a través de ilustraciones, cómo los edificios y casas se han vuelto cada vez más parecidos a prisiones, mientras que las prisiones son "naturalizadas arquitectónicamente" (ibíd.:256). La tapa del libro es una fotografía del Centro de Detención Metropolitano en el centro de Los Ángeles, al cual denomina "una Bastilla posmoderna" (ibíd.:257). En cuanto al punto de que edificios y casas se vuelven cada vez más parecidos a prisiones, podríamos retomar los abundantes estudios etnográficos de comunidades cerradas. Setha Low (2010) ha documentado el incremento de tales comunidades en los Estados Unidos. Sabemos que cada vez más personas se mudan hacia barrios cerrados para sentirse más seguras del "crimen", aunque Low muestra etnográficamente que la gente en esas comunidades en realidad se siente menos y no más segura: "Vivir detrás de rejas refuerza la percepción de que la gente que vive afuera es peligrosa o mala. Esta separación social siempre ha existido, pero los muros y las rejas exacerban las distinciones sociales" (ibíd.:35). Teresa Caldeira (2001) ha emprendido un estudio de barrios cerrados en São Paulo, Brasil. Ella define a São Paulo como una "ciudad de muros" y al igual que Mike Davis, escribe sobre -e ilustra con fotografías- una "estética de la seguridad" que se ha expandido por la ciudad (ibíd.:291).

¿Cuál es la lógica que conecta la proliferación de prisiones y la construcción de un entorno a partir de modelos que copian a las prisiones, a la teoría económica neoliberal? Como con todo lo demás sobre el neoliberalismo, hay una lógica de arriba hacia abajo y otra de abajo hacia arriba. Desde el punto de vista del estado y otras entidades de gobernanza, la proliferación de prisiones es parte de la guerra contra los pobres y los que se encuentran racialmente marginados: a través de una lógica que consiste en culpar-a-la-víctima, son estereotipados como sujetos neoliberales irresponsables (si fueran responsables, no serían pobres) y encarcelados a la más mínima señal de mal comportamiento, y a veces sin ningún tipo de señal. Desde abajo hacia arriba -esto es, desde el punto de vista nativo-, del otro lado, la lógica emerge de la atmósfera más amplia de inseguridad alimentada por el riesgo en los trabajos y su cultura asociada de precariedad. La precariedad de la vida bajo el neoliberalismo se proyecta hacia afuera como una amenaza de los pobres u otros racializados; la emergencia histórica de las comunidades cerradas corresponde, como muchos observadores han notado (p. ej., Caldeira 2001; Ortner 2013a) no al 
incremento en la tasa de crímenes - de hecho la tasa de crímenes violentos ha estado en declinación en todo el periodo- sino al despliegue de la economía neoliberal. ${ }^{6} \mathrm{Y}$ como una coda a esta historia, notamos que las prisiones en sí mismas no escapan a la neoliberalizacion. Muchas prisiones en Estados Unidos fueron privatizadas y son ahora administradas como negocios para obtener ganancias. ${ }^{7}$

Finalmente, se puede ver que la gobernanza punitiva abarca la racionalidad económica discutida en la sección previa, y podemos ver esto también etnográficamente. Noté antes que, a pesar de que los recortes en la fuerza laboral norteamericana fueran representados como una especie de mal económico necesario en relación con la competencia global, de hecho a veces parecían tener una motivación más irracional y casi cruel, que discutí en términos de los varios ejemplos de la guerra contra los pobres. Pero las irracionalidades punitivas de los recortes también pueden ser vistas en el tope de la escala de las ocupaciones, como por ejemplo en el estudio de Karen Ho (2009) sobre los banqueros de Wall Street. Ho presenta un retrato de los bancos de inversión de Wall Street implicándose en ciclos de contratos y despidos que solo están relacionados en parte con las fluctuaciones del mercado. Por el contrario, la idea de inseguridad laboral está profundamente normalizada y es incluso valorada como una manera de que las empresas parezcan flexibles y dinámicas.

De hecho, los trabajadores a lo largo y ancho de la nueva economía han sido forzados a adaptarse a una vida en la que los trabajos son precarios, el desempleo es familiar y pasar largos periodos de tiempo como "contratados independientes" es normal. Richard Sennett (1998) ha llamado a esto la lógica de "nada a largo plazo" [no long term]: todo trabajo hoy en día es profundamente inseguro y puede ser cortado en cualquier momento. Y como con la persona auto-administrada y la estética de la prisión, esto ha sido generalizado a la cultura en su totalidad. Así, David Harvey comienza su breve historia del neoliberalismo con una cita a este respecto de Jean François Lyotard (1984) sobre la condición posmoderna "como aquella en la que el 'contrato temporal' suplanta 'las instituciones permanentes

\footnotetext{
'La propagación de comunidades cerradas también puede ser interpretada en relación con la demanda por "privatización" en la lógica económica neoliberal. Sin embargo, el término "privatización" en el contexto económico no trata sobre "privacidad" personal, sino sobre vender bienes públicos a negocios privados. Aun así, en cierta manera, el resultado es el mismo: cuando la propiedad pública es vendida o cuando las personas se retiran a comunidades cerradas, el resultado es el debilitamiento de la esfera pública.

${ }^{7}$ Esta es de por sí una triste historia, pero ha llegado al extremo en una -ahora famosa- trama de comisiones ilegales en Pensilvania, donde dos jueces se confabularon con los propietarios de una instalación privada de detención juvenil local para enviar a cientos de niños a prisión, de modo que los dueños/carceleros pudieran cobrar las tasas per cápita. (Moore 2009; Ecenbarger 2012).
}

en los dominios profesionales, emocionales, sexuales, culturales, familiares e internacionales, como así también en los asuntos políticos'" (Harvey 2005:4).

\section{Antropologías de lo bueno}

He brindado un panorama de lo que llamé el giro hacia la "antropología oscura" - a teorías de poder ubicuo y desigualdad, y a estudios etnográficos de, en el mejor de los casos, inseguridad económica y gubernamentalidad punitiva. Pero el giro hacia la antropología oscura no ha pasado desapercibido y, de hecho, ha provocado reacciones fuertes en algunos espacios. Notando el "marcado incremento en el trabajo antropológico que analiza experiencias de violencia y crueldad", por ejemplo, Tobias Kelly pregunta “ ¿Hasta qué punto la etnografía del sufrimiento no se ha vuelto una cuasi pornografía voyerista?" (2013:213). De hecho, hay varios tipos de trabajos que pueden ser considerados como una forma de resistencia al giro oscuro; haré una revisión breve sobre ellos aquí.

En un influyente artículo titulado "Beyond the suffering subject: Toward an anthropology of the good", Joel Robbins sostiene que "el sujeto sufriente... que vive en el dolor, en la pobreza o bajo condiciones de violencia u opresión se ubica a menudo en el centro del trabajo antropológico actual" (2013:448). Robbins apunta a establecer, en su lugar, "una antropología de lo bueno... enfocada en tópicos tales como valor, moralidad, bienestar, imaginación, empatía, cuidado, el don, esperanza, tiempo y cambio" (ibíd.).

Entre esos términos, "bienestar" se ha vuelto uno de los mayores focos de investigación. Por ejemplo, The good life: aspiration, dignity and the anthropology of wellbeing de Edward Fisher (2014), es un estudio sobre las ideas de bienestar entre alemanes de clase media y productores de café guatemaltecos. Tal como la "antropología de lo bueno" de Robbins, Fischer considera su libro como parte de -y contribuyendo a- lo que él llama "una antropología positiva" (ibíd.:17). Cercana a la idea de bienestar está la de "felicidad". Al año siguiente, HAU publicó un número especial titulado "Happiness: Horizons of purpose" (Vol. 5, No. 3), que consistía en una serie de artículos sobre la felicidad en un amplio rango de casos etnográficos. En la introducción, los editores se refieren "al reciente 'giro hacia la felicidad' en las ciencias sociales" (Walker and Kavedžija 2015:2), y llaman a los antropólogos a contribuir más con esta tendencia. Ellos especulan acerca de las razones de la relativa ausencia de esta área de trabajo en antropología: para los antropólogos "hay una cierta sospecha sobre la felicidad como una preocupación esencialmente burguesa, crecientemente asociada con una agenda neoliberal y potencialmente contraria a políticas emancipatorias" (ibíd.:4); y que "la disciplina frecuentemente ha gravitado hacia formas de experiencia humana más 'negativas', tales como el sufrimiento, el dolor o la pobreza" (ibíd.). Así, ellos reclaman una antropología más positiva, aunque 
debe decirse que las discusiones en los artículos específicos muestran a la felicidad como un fenómeno mucho más complejo y ambivalente que el que la propia palabra tiende a evocar, al menos en inglés americano.

Estos trabajos son interesantes e importantes. Coincido con que es importante indagar de cerca "sobre lo que da un sentido de propósito o dirección en la vida, o cómo las personas buscan la mejor manera de vivir -aun en circunstancias terribles y hostiles" (ibíd.:17), y que la "felicidad" parece proveer una entrada a este tema. También coincido en que los antropólogos casi siempre están mejor preparados para hacer este tipo de preguntas y pensar profundamente sobre las respuestas que los encuestadores, que son quienes controlan gran parte de la pesquisa sobre la felicidad hoy en día. Pero confieso que me sorprendió el "giro hacia la felicidad" en medio de toda la oscuridad discutida en la sección previa de este artículo. Reflexionando, sin embargo, tiene sentido precisamente como una reacción a ese trabajo.

La otra gran área de trabajo que responde -al menos en parte- al giro oscuro es la producción sobre moralidad y ética. Michael Lambek abre su importante colección Ordinary Ethics con la siguiente afirmación:

"Los etnógrafos comúnmente descubren que las personas que encuentran intentan hacer lo que consideran como correcto o bueno, y están siendo evaluadas de acuerdo con criterios de lo que es correcto y lo que está bien, o están en algún debate sobre lo que constituye el bien humano. Sin embargo, la teoría antropológica tiende a ignorar todo esto en favor de análisis que enfatizan la estructura, el poder y el interés" (2010a:1)

El autor presenta una lista de temas cubiertos en la compilación que fue quizás la inspiración para la lista de Robbins, citada anteriormente: "libertad, juicio, responsabilidad, dignidad, autoformación [self-fashioning], cuidado, empatía, carácter, virtud, verdad, razonamiento, justicia y la buena vida para la humanidad" (ibíd.:6).

La antropología de la moralidad y la ética es en la actualidad un subcampo de investigación y teorización grande, extenso y algo controversial, que incluye tanto un lado más social, proveniente al menos en parte del trabajo de Durkheim, como uno más individual, construido mayormente en oposición al paradigma durkheimiano. He notado lo primero en relación con mi punto anterior acerca de que la posición de Durkheim entre los ancestros teóricos se ha desvanecido mucho. Durkheim ciertamente se desvaneció con el paso del funcionalismo y de la visión holística de la sociedad que prevaleció en la antropología a comienzos de mediados del siglo XX. También se desvaneció con relación al ascenso de teorías del poder, de la dominación y de la explotación sobre las cuales tenía poco para decir. Por otro lado, como teórico de la sociedad como universo moral, ha adquirido una relevancia renovada en este conjunto de trabajos, tanto a favor como en contra (Lambek 2010b; Yan 2011; Fassin 2014).

El movimiento de la moralidad/ética presenta otro importante complemento al giro oscuro en la teoría antropológica y en el trabajo etnográfico discutido en la primera parte de este artículo. El foco en los intentos de actores reales de lidiar con dilemas morales y realizar elecciones éticas puede ser visto como un contrapeso positivo y humano a la oscuridad del trabajo sobre la opresión neoliberal y el constreñimiento gubernamental. Similarmente, el foco en temas como cuidado, amor, empatía, responsabilidad, y sobre tratar de hacer lo correcto aún si se fracasa, es un contrapunto refrescante y edificante a una dieta estricta del Foucault temprano, en la cual ninguna buena acción queda sin castigar y en la que cada acción potencialmente positiva simplemente magnifica las redes de poder en las que vivimos.

Mi única preocupación acerca de este -de nuevo, valorable- trabajo, incluyendo tanto la versión más psicológica/médica con su énfasis en la búsqueda de la felicidad y/o el bienestar, y la versión más ética/moral con su énfasis en (la búsqueda de) la virtud y lo bueno, es la marcada línea que a veces es trazada entre éste y el trabajo sobre poder, desigualdad y violencia discutido previamente. En lugar de presentar una relación opuesta entre los dos, sería útil aquí considerar obras que apuntan a integrarlos. Un buen punto de partida sería el ensayo de Veena Das en el volumen de Lambek titulado "Engaging the life of the other: Love and everyday life" (2010). El artículo trata sobre el matrimonio de un hombre hindú y una mujer musulmana en un barrio de bajos ingresos en Delhi, y la manera en que esto lentamente se resuelve a la sombra de una historia larga y continua de violencia y enemistad hindu-musulmana. A lo largo del artículo, Das avanza y retrocede entre, por un lado, la construcción de relaciones cotidianas entre la novia, el novio y sus respectivas familias, y por otro, las implicaciones políticas mayores y el potencial para la violencia que rodea a los matrimonios entre castas y religiones. Ella insta a "prestar atención a la manera en la cual el esfuerzo moral se muestra en los trabajos cotidianos de cuidar al otro, aun en contextos donde el antagonismo mutuo define la relación" (ibíd.:398-99).

Didier Fassin también ha dedicado un extenso trabajo a explorar las complejidades de la ética en el contexto de situaciones políticas de desigualdad y violencia. En su conferencia/ensayo "Troubled waters: At the confluence of ethics and politics" (2015), por ejemplo, considera tres casos en diferentes niveles de escala: las intervenciones europeas y norteamericanas en Libia bajo el principio de la "responsabilidad de proteger" de las Naciones Unidas; la respuesta nacional francesa a los ataques terroristas en Charlie Hebdo y en un supermercado kosher en Paris; y las maneras en las cuales la policía trata a los jóvenes en las zonas densamente pobladas por inmigrantes y minorías de los suburbios parisinos. Explorando la "diferenciación heurística entre la ética de la convicción y la ética de la 
responsabilidad" de Weber, argumenta que esto "necesita ser evaluado en cada contexto histórico y político, considerando más específicamente las relaciones de poder y los juegos de poder en los que se insertan" (ibíd.:195).

En suma, estoy de acuerdo con los investigadores de la buena vida en que el estudio del poder y la desigualdad, y del daño que hacen, no puede ser todo en antropología. También concuerdo con los estudiosos de la moralidad y la ética en que es importante observar el cuidado y las dimensiones éticas de la vida humana, porque ¿cuál es el punto de oponerse al neoliberalismo si no podemos imaginar mejores modos de vida y mejores futuros? ¿Cómo se puede ser realista sobre las desagradables realidades del mundo en la actualidad y esperanzado sobre las posibilidades de cambiarlas? En la sección final del artículo me oriento a un tipo diferente de antropología de lo bueno: la antropología de la crítica, de la resistencia y del activismo.

\section{El regreso de la resistencia}

Uso el término "resistencia" para cubrir diversos modos de compromiso (antropológico) con temas políticos: discusiones de teoría crítica; estudios de etnografía crítica; estudio de movimientos políticos de todo tipo; antropología activista; y más. En esta sección voy a tratar algo de esta variedad e inventariar la amplia gama de trabajos importantes en esta área, en el mismo periodo en el cual la "antropología oscura" ha estado en ascenso.

La antropología de la resistencia -en una forma u otraha tenido sus altibajos. Hubo una ráfaga de interés etnográfico y teórico en 1980 (Comaroff 1985; Ong 1987; Scott 1985, 1990). Pero para el momento en que publiqué un artículo sobre ese asunto a mediados de los 90 ya había una cierta reacción, si no contra el concepto, al menos contra el término (Ortner 1995). Dimitrios Theofossopoulos ha argumentado que la resistencia fue "patologizada" y "exotizada", y que perdió su legitimidad como objeto de estudio (2014:415). Por qué y cómo esta "resistencia a la resistencia" tomó forma es poco claro, y probablemente varía según los diferentes contextos de investigación. La cuestión es que el concepto se extendió demasiado en su significación - Rabinowitz se refiere a su "debilidad teórica" (2014:476)- y también fue usado en exceso. Ello puede ser cierto, pero para los propósitos presentes señalaría un conjunto diferente de factores: el mundo real en los 90 se estaba volviendo más sombrío, mientras las promesas de los movimientos sociales de los 60 y 70 empezaban a desvanecerse, y los jóvenes nacidos entre 1960 y 1970 empezaban a experimentar el comienzo del fin del "sueño americano" (Ortner 1998, 2013a). "Resistencia" puede haber empezado a parecer una perspectiva menos realista e incluso absurda, mientras la "gubernamentalidad", las miles de formas en las que personas y poblaciones son moldeadas y reguladas, adquirió una relevancia creciente en las condiciones de vida del mundo real. Tal vez no sea un accidente que este periodo también haya visto el ascenso de las teorías de la "posmodernidad", incluyendo entre otras cosas el "fin de las grandes narrativas" como aquellas de la resistencia y la revolución (Jameson 1984; Lyotard 1984). Sin especular mucho más sobre las conexiones con el mundo real, parece claro que la antropología de la resistencia, en una gran variedad de formas, está de regreso.

Podríamos empezar con la distinción de Charles Hale entre "investigación activista" y "crítica cultural" (2006). Por investigación activista se refiere a la investigación realizada en el transcurso de comprometerse personalmente en la lucha política bajo estudio; por "crítica cultural" se refiere al trabajo etnográfico que profesa solidaridad política con la causa estudiada, pero en el cual el etnógrafo no está directamente involucrado en la lucha política en cuestión. Expresa un "rechazo inflexible" a aceptar la crítica cultural como "un lugar de descanso de la pesquisa y escritura antropológica" (ibíd.:97). Sin embargo, como veremos, ambos tipos de trabajo están floreciendo en la antropología actual y preferiría ver a estos -y a los demás- como parte de un paquete más amplio de trabajo antropológico crítico en el que los diversos elementos se sostienen y se enriquecen unos a otros.

Distinguiría al menos tres grandes áreas en las que se está realizando un trabajo importante: (1) crítica cultural (pace Hale), que incluye escritos etnográficos críticos sobre condiciones de desigualdad, poder y violencia en varias partes del mundo; (2) una serie de trabajos, en su mayoría teóricos, que apuntan a repensar el capitalismo como sistema y finalmente (3) un conjunto de trabajos sobre movimientos sociales que han tomado forma en el periodo neoliberal. Diré algunas palabras sobre cada uno.

Bajo la rúbrica de "crítica cultural", señalaría los estudios empíricos críticos reseñados previamente en este artículo, incluyendo tanto estudios etnohistóricos en el caso del colonialismo, y estudios más estrictamente etnográficos. Los últimos incluyen etnografías de comunidades impactadas económicamente por el neoliberalismo, mostrando las diversas maneras en las cuales individuos y comunidades experimentaron las consecuencias de la desindustrialización, la globalización, y la pérdida de trabajos y oportunidades. Este grupo también incluye estudios de negocios e industrias que se han organizado para aprovecharse de este tipo de pérdidas. Más allá de lo económico, consideramos los estudios de la gubernamentalidad neoliberal, incluyendo la proliferación de las prisiones, el crecimiento del "estado carcelario" y la expansión de la "estética de la prisión". Traigo todo esto de vuelta aquí porque es importante reconocer estos trabajos no como ejemplos de una "pornografía de la miseria" (por más sombríos que sean), sino como ejemplos de un género etnográfico crítico nuevo e importante, que arroja luz sobre el mundo en que vivimos en la actualidad, revelando su funcionamiento interno.

También incluyo en este grupo un conjunto de trabajos 
críticos que se realizó en la amplia zona de la antropología de los medios de comunicación. Por ejemplo, la compilación clave Media worlds: Anthropology on new terrain (2002) editada por Faye D. Ginsburg, Lila AbuLughod y Brian Larkin, incluye ensayos sobre este tema que abarcan desde el uso de los medios en las políticas nativas, pasando por el uso de los medios por los estados-nación en proyectos culturales e ideológicos de gran escala, hasta la circulación sobre el terreno de las tecnologías mediáticas entre grupos diaspóricos y más. También incluiría aquí mi propio estudio sobre el mundo del cine independiente, que emplea explícitamente el concepto de crítica cultural para pensar cómo los norteamericanos y otros cineastas independientes usan las películas para lidiar con las nuevas condiciones de vida en el orden neoliberal (Ortner 2013a).

Bajo la siguiente rúbrica de "repensando el capitalismo" hay en primer lugar un conjunto de trabajos teóricos recientes por fuera de la antropología, de los cuales se pueden mencionar aquí algunos ejemplos: Empire (2000) del crítico literario Michael Hardt y el sociólogo Antonio Negri, sostiene que debemos entender el mundo actual en términos de nuevas configuraciones de dinero, poder y leyes que son casi completamente post-nacionales; The new spirit of capitalism (2005) de los sociólogos Luc Boltanski y Eve Chiapello analiza las maneras en las que el capitalismo en tanto sistema ha sido y continúa siendo susceptible de crítica interna; y Rethinking capitalist development: Primitive accumulation, governmentality, and post-colonial capitalism (2007) del economista Kalyan Sanyal explora las implicaciones de la creciente exclusión de una vasta cantidad de personas pobres de las estructuras principales de las economías capitalistas.

Los antropólogos también están "repensando el capitalismo" y de manera más general repensando las "economías" en el mundo contemporáneo. Este trabajo incluye una compilación importante de Keith Hart, JeanLouis Laville y Antonio David Cattani titulada The human economy (2010), que sostiene que debemos observar el mundo real de las prácticas económicas variadas en lugar de simplemente el "capitalismo" como una entidad monolítica, e incluye una gran cantidad de artículos sobre -entre otras cosas- "economías con un rostro humano" y "políticas morales". Una línea de pensamiento similar se encuentra en el trabajo del grupo "Generating capitalism" (Bear et al. 2015). Este grupo ha producido "un manifiesto feminista para el estudio del capitalismo" que sostiene la importancia de reconocer que el capitalismo no se trata solo de la "economía", sino que todos los lugares de la producción de valor son internos al capitalismo en sí. Ellos apuntan a construir "la crítica feminista de Marx, que afirmaba que el parentesco, la personalidad, el espacio doméstico y la reproducción social residen firmemente dentro de creaciones capitalistas de valor" (ibíd.). Etnografías inspiradas por estas nuevas perspectivas han empezado a aparecer. Ejemplos recientes en esta categoría incluirían Give man a fish: Reflections on the new politics of distribution de James Ferguson (2015) y Dream Zones:
Anticipating capitalism and development in India de Jamie Cross (2014). Estos y otros trabajos buscan expandir y quebrar una concepción monolítica del capitalismo, y hacer lugar para un espectro más amplio y diverso de proyectos sociales y políticos.

Finalmente, vuelvo a la antropología de la resistencia en su forma clásica: esto es, el estudio de los movimientos sociales. Comienzo con algunas pocas palabras sobre Pierre Bourdieu, quien se volvió activo en el movimiento antiglobalización y antineoliberal, y cuyo libro titulado Acts of resistance: Against the tyranny of the market (1998) se encuentra entre las publicaciones más tempranas de un antropólogo-como-activista. ${ }^{8}$ Al final de uno de los primeros artículos sobre el estado de la antropología (1984), yo sostenía que el trabajo teórico de Bourdieu, entre otros, constituía una ruptura importante con las anteriores teorías que se basaban en las restricciones de la vida social, insistiendo tal como hizo en la propuesta de que la sociedad y la cultura son producidas y reproducidas a través de la práctica humana. Aunque Bourdieu en general enfatizaba las maneras en que las prácticas sociales tendían a reproducir el mundo en lugar de cambiarlo (sin embargo, véase Gorski 2013; Ortner 2013b sobre Bourdieu y la historia), las posibilidades transformadoras de lo que entonces llamaba la "teoría de la práctica" fueron inmediatamente evidentes para mí. En sus términos más simples, si hacemos el mundo a través de la práctica social, podemos deshacerlo y rehacerlo a través de la práctica social. O como David Graeber escribió más tarde, "La verdad última y oculta del mundo es que es algo que nosotros hacemos y que fácilmente podríamos hacer de otro modo" (2009:514). No estoy segura acerca de la parte de "fácilmente", pero en cualquier caso el trabajo de Bourdieu en particular y de la teoría de la práctica en general (p. ej., Sahlins 1981) debe ser claramente visto como los cimientos fundacionales del trabajo en y sobre la resistencia.

De más está decir que hay todo tipo de movimientos sociales, a favor y contra todo tipo de cosas: feminismo(s), movimientos ambientales, movimientos de justicia racial, derechos indígenas, derechos de discapacitados y mucho más. Aquí, manteniendo la temática general de este artículo, me concentraré específicamente en la proliferación de trabajos sobre movimientos antiglobalización y antineoliberalismo. El trabajo en esta área empezó a florecer en la primera década del siglo veintiuno. (Para una reseña excelente, véase Kurik s.f.) Varias compilaciones de ensayos han aparecido, incluyendo Engaging contradictions: Theory, politics and methods of activist scholarship de Charles Hale (2008), Insurgent encounters: Transnational activism, ethnography, and the political de Jeffrey Juris y Alex Khasnabish (2013) y Rethinking resistance in the 21st century: History and anthropology de Dimitrios Theodossopoulos (2014).

\footnotetext{
${ }^{8}$ Sé, por supuesto, que Bourdieu es técnicamente un sociólogo,
} pero también ha sido una figura importante en la antropología. 
Todas éstas contienen múltiples ensayos de académicos en formación o profesionales sobre movimientos de resistencia en diversas partes del mundo. Ellos ofrecen un sentido efectivo de la explosión del interés en tales trabajos, la diversidad de temas hacia los cuales los movimientos se dirigen en la actualidad, y la variedad de problemas conectados con hacer investigación etnográfica en estos contextos.

Asimismo, tenemos algunos pocos estudios etnográficos en profundidad sobre varios movimientos, escritos desde el punto de vista de una participación profunda del antropólogo. Networkin futures: The movements against corporate globalization (2008) es un estudio de una amplia red de activistas situada en Barcelona pero que actúa en muchos lugares a lo largo y ancho del mundo, incluyendo la movilización exitosa en Seattle en 1999 contra las reuniones de World Trade Organization. Direct action: An ethnography (2009) de David Graeber es un estudio sobre el trabajo de un grupo llamado Direct Action Network, con sede en la ciudad de Nueva York, mientras desarrollaban y llevaban adelante una gran campaña de acción directa contra la Cumbre de las Américas en la ciudad de Québec en 2001. Finalmente, tenemos varios ensayos de Arjun Appadurai (2013) sobre su trabajo en Bombay, India, con una organización transnacional llamada Schak/Slum Dwellers International, que trabaja en temas de vivienda para los pobres.

Lo que es nuevo en muchos de estos de estudios es el giro hacia una "antropología activista": esto es, hacia un trabajo en el cual los propios antropólogos no están simplemente haciendo la investigación, sino que están directamente involucrados en los movimientos. Los antropólogos que estudiaban movimientos sociales en el pasado a menudo fueron llevados a involucrarse en cierto nivel, pero en muchos casos ahora el antropólogo es tanto un participante completo como un observador. Esto ha producido una literatura vívida y fascinante sobre las contradicciones y desafíos experimentados durante el trabajo de campo, pero también reflexiones más amplias sobre lo que toda esta resistencia esperaría lograr realmente. Para un ejemplo sobre este punto, observemos las respuestas antropológicas a Occupy Wall Street en 2011. Los eventos en Nueva York produjeron una avalancha de publicaciones antropológicas comenzando con el número de Mayo de 2012 de American Ethnologist. La revista mostraba una foto de los manifestantes de Occupy en la tapa, como también dos artículos y un comentario sobre los hechos (Juris 2012; Razsa y Kurnik 2012). En otra revista, Hannah Appel publicó notas etnográficas sobre la ocupación (2012) y en 2014 publicó un artículo, "Occupy Wall Street and the economic imagination". En este caso (y en los varios referidos previamente), la participación/observación del etnógrafo no provee simplemente una etnografía "densa" de las demostraciones. Aunque ciertamente lo hace, también brinda aperturas hacia reflexiones teóricas mucho más amplias -sobre la naturaleza de la democracia y la participación política, de manera general y, en este caso, sobre lo que Appel llama "la imaginación económica".

Para otro ejemplo, volvamos al trabajo de Arjun Appadurai sobre los grupos con sede en India que forman parte de la red de Shack/Slum Dwellers Internacional. Las personas involucradas se encuentran entre los más pobres de los pobres, las masas de personas a quienes Kalyan Sanyal describe como situadas en el fondo del capitalismo y a la vez producidas continuamente por fuera del mismo. Appadurai describe una cantidad de acciones en las cuales el grupo estaba involucrado y también una iniciativa que él desarrolló colaborativamente con el grupo que incluía un entrenamiento en métodos de investigación básicos, con el eslogan "Documentation is Intervention" (2013:280). Appadurai usa todo ello para reflexionar más ampliamente sobre la significación del activismo tanto para las personas involucradas como para la antropología contemporánea. Apuntando directamente a la oscuridad del largo momento contemporáneo, él plantea un contraste entre lo que llama una ética de la probabilidad y una ética de la posibilidad. La ética de la probabilidad apuesta a lo que llamamos el lado oscuro del mundo y de la antropología (Appadurai apunta específicamente al trabajo de Naomi Klein sobre el "capitalismo de desastre"), mientras que la ética de la posibilidad está basada en "aquellas maneras de pensar, sentir y actuar que amplían los horizontes de la esperanza" (ibíd.:295). Appadurai llama a los antropólogos "a ser mediadores, facilitadores y promotores de la ética de la posibilidad, la cual puede ofrecer una plataforma más inclusiva para mejorar la calidad de vida del planeta y puede albergar una pluralidad de visiones sobre la buena vida" (ibíd.:299).

\section{Breves conclusiones}

Antes de avanzar propiamente hacia las conclusiones, vale la pena repetir mi reconocimiento previo de que este artículo sobre el neoliberalismo y la antropología no fue escrito desde ninguna otra perspectiva que no fuera la norteamericana. Otros tendrán visiones distintas, ya sea porque las cosas son genuinamente diferentes en otros lugares o porque las personas siempre tendrán, es claro, distintas visiones. Tampoco he intentado sugerir que el neoliberalismo es la causa de todas las cosas malas en el mundo y hay varios otros tipos de ejes de desigualdad, conflicto y violencia -género, raza, etnicidad, religión- que tienen sus propias dinámicas, aun cuando se articulan con un orden neoliberal agresivo.

Este artículo comenzó con el giro a la "antropología oscura", argumentando que desde 1980, aproximadamente, la emergencia del capitalismo neoliberal tuvo efectos profundos en el campo, en los niveles tanto de la teoría como de la etnografía. Marx (incluyendo varios post y neo-marxismos) y Foucault, con sus teorías que destacan la explotación, la desigualdad y el funcionamiento del poder, han llegado a dominar el campo teóricamente. Además, ha emergido una gran cantidad de estudios etnográficos que observaron el impacto del neoliberalismo como sistema 
económico y sistema de gubernamentalidad. Como la teoría, la etnografía es a menudo oscura, destacando la naturaleza dura, violenta y punitiva del neoliberalismo, y la depresión y desesperanza en las que están envueltas las personas bajo regímenes neoliberales.

En la siguiente sección observé el trabajo sobre las "antropologías de lo bueno" con la idea de que "lo bueno" incluye tanto el bienestar ("la buena vida", "felicidad") como la moralidad y la ética. Este trabajo configura un contrapunto importante a las antropologías oscuras discutidas en la primera parte del artículo, pero su relación con esas obras puede ser a menudo problemática. En algunos casos, los autores colocan el trabajo sobre lo bueno en oposición al trabajo sobre la opresión y la desigualdad (desestimado como "porno de la miseria"), en otros casos simplemente ignoran los contextos más amplios de poder y desigualdad en juego. En respuesta a eso, traté de destacar la importancia de mantener estos dos tipos de trabajo o, más ampliamente, estas dos perspectivas en activa interacción la una con la otra, en lugar de en oposición. Porque la violencia del poder y la desigualdad no es simplemente fuerza física y/o privación, sino también las maneras en las cuales limita y deforma proyectos de lo que Veena Das ha llamado "lo cotidiano": proyectos de cuidado y amor, de felicidad y de buena vida.

En la sección final del artículo observé el nuevo trabajo emergente sobre resistencia y activismo. Usé el término "resistencia" ampliamente para incluir distintos tipos de trabajo: etnografía crítica, incluyendo etnografía crítica de los medios de comunicación, agrupada en la categoría de "crítica cultural"; nuevo trabajo teórico y etnográfico apuntando a repensar la categoría aparentemente monolítica de "capitalismo", y abriendo así nuevas visiones de economías políticas alternativas; y finalmente, el estudio etnográfico de los movimientos sociales, incluyendo un amplio subconjunto en el cual el antropólogo es un participante activo. Las descripciones etnográficamente densas de la resistencia en todas estas categorías son importantes no solo para entender la extraordinaria variedad de maneras creativas en las que se construyen desafíos al orden existente, sino también para entender las visiones alternativas del futuro incorporadas en tales movimientos.

Quiero concluir señalando que la antropología de la resistencia, al menos tal como la defino aquí, incluye la "crítica cultural" -esto es, el estudio crítico del orden existente- y los estudios que enfatizan el pensamiento sobre futuros políticos y económicos alternativos (tanto "repensando el capitalismo", como los movimientos sociales). La crítica cultural tiende a ubicarse en el lado oscuro, destacando lo que Arjun Appadurai llama de "ética de la probabilidad", mientras que estos otros tipos de trabajo están al menos parcialmente orientados hacia visualizar alternativas más positivas, encarnando lo que Appadurai llama de "ética de la posibilidad". Parece probable que la antropología siempre necesitará de los dos.
Es, entonces, apropiado terminar con una frase famosa de Antonio Gramsci: "pesimismo del intelecto, optimismo de la voluntad".

\section{Agradecimientos}

Agradezco profundamente a Timothy D. Taylor por las varias lecturas de este ensayo y por sus comentarios siempre perspicaces. Por los excelentes comentarios y devoluciones, también agradezco a mi anfitriona Angelica Wehrli en la Universidad de Lucerna y en otros lugares en Suiza; mis anfitriones en la London School for Economics, Rita Astuti y Charles Stafford; los estudiantes de mi seminario de neoliberalismo en la Universidad de California, Los Ángeles; y mis camaradas en el indispensable grupo de interés en UCLA "Culture/Power/Social Change" (CPSC). Por cada comentario servicial y valorable, también extiendo mi agradecimiento a los siguientes colegas, amigos y lectores: Hannah Appel, Rogers Brubaker, James Laidlaw, Michael Lambek, Jonathan Parry, Danilyn Rutherford, y Jason Throop. Finalmente, gracias a Justin Dyer por su pensativa y cuidadosa edición, y al final pero ciertamente no por último a Giovanni da Col por su agudeza, energía y entusiasmo y su amable ayuda como editor. Todos estos comentarios increíblemente inteligentes y perceptivos han elevado el nivel de este artículo inconmensurablemente.

\section{Bibliografía}

Adams, V. (2013). Markets of sorrow, labors of faith: New Orleans in the wake of Katrina. Durham, NC: Duke University Press.

Allison, A. (2013). Precarious Japan. Durham, NC: Duke University Press. American Ethnologist. 2012. "Occupy movements: AE forum," Vol. 39, No. 2, 238-79.

Appadurai, A. (2013). The future as cultural fact: Essays on the global condition. London and New York: Verso.

Appel, H. (2012). "Dispatches from an occupation: Ethnographic notes from occupied Wall Street." Social Text Online Journal. http://www.socialtextjournal.org/blog/topics/dispatches-froman-occupation/.

Appel, H. (2012). (2014). "Occupy Wall Street and the economic imagination." Cultural Anthropology. 29 (4): 602-25. http://www.culanth.org/articles/753-occupy-wall-street-andtheeconomic- imagination.

Asad, T., Ed. (1973). Anthropology and the colonial encounter. Atlantic Highlands, NJ: Humanities Press.

Bear, L., K. Ho, A. Tsing \& S. Yanagisako. (2015). "Generating capitalism" and "Gens: A feminist manifesto for the study of capitalism." Fieldsights: Theorizing the contemporary, Cultural Anthropology Online, March 30. http://www.culanth.org/ fieldsights/652-gens-a-feminist-manifesto-for-the-study-ofcapitalism Accessed 4/3/16.

Berlant, L. (2011). Cruel optimism. Durham, NC: Duke University Press.

Boltanski, L., \& E. Chiapello. (2005). The new spirit of capitalism. 
Translated by Gregory Elliott. London and New York: Verso. Bourdieu, P. (1998). Acts of resistance: Against the tyranny of the market. Translated by R. Nice. New York: The New Press.

Bourdieu, P, et al. 1993. The weight of the world: Social suffering in contemporary society. Translated by P. P. Ferguson, et al. Stanford, CA: Stanford University Press.

Brenner, R. (2006). The economics of global turbulence: The advanced capitalist economies from long boom to long downturn, 1945-2005. London and New York: Verso.

Brown, W. (2003). "Neo-liberalism and the End of Liberal Democracy." Theory and Event 7 (1). doi: 10.1353/tae.2003.0020.

Burchell, G., C. Gordon \& P. Miller, Eds. (1991). The Foucault effect: Studies in governmentality. Chicago: University of Chicago Press.

Caldeira, T. P. R. 2001. City of walls: Crime, segregation, and citizenship in São Paulo. Berkeley, CA: University of California Press.

Clifford, J. and G. E. Marcus, Eds. (1986). Writing culture: The poetics and politics of ethnography. Berkeley, CA: University of California Press.

Cohn, B. S. (1996). Colonialism and its forms of knowledge: The British in India. Princeton, NJ: Princeton University Press.

Collins, J. (2010). "The age of Wal-Mart." In The insecure American: How we got here and what we should do about it, edited by Hugh Gusterson and Catherine Besteman, 97-112. Berkeley, CA: University of California Press.

Comaroff, J. (1985). Body of power, spirit of resistance: The culture and history of a South African people. Chicago: University of Chicago Press.

Comaroff, J. \& J. Comaroff. (1991). Of revelation and revolution, volume 1: Christianity, colonialism, and consciousness in South Africa. Chicago: University of Chicago Press.

Comaroff, J. \& J. Comaroff. (1997). Of revelation and revolution, volume 2: The dialectics of modernity on a South African frontier. Chicago: University of Chicago Press.

Costa, L. \& C. Fausto. 2010. "The return of the animists: Recent studies of Amazonian ontologies." Religion and Society: Advances in Research 1: 89-109.

Cross, J. (2014). Dream zones: Anticipating capitalism and development in India. London: Pluto Press.

da Col, G. and D. Graeber. (2011). "Foreword: The return of ethnographic theory." Hau: Journal of Ethnographic Theory 1 (1): vi-xxxv.

Das, V. (2010). "Engaging the life of the other: Love and everyday life." In Ordinary ethics: Anthropology, language, and action, edited by Michael Lambek, 376-99. New York: Fordham University Press.

Davis, M. (1992). City of quartz: Excavating the future in Los Angeles. New York: Vintage Books.
Dirks, N. B. (2001). Castes of mind: Colonialism and the making of modern India. Princeton, NJ: Princeton University Press.

Dudley, K. M. (1994). The end of the line: Lost jobs, lives in postindustrial America. Chicago: University of Chicago Press.

Duménil, G., \& D. Lévy. (2004). Capital resurgent: Roots of the neoliberal revolution. Translated by Derek Jeffers. Cambridge, MA: Harvard University Press.

Ecenbarger, W. (2012). Kids for cash: Two judges, thousands of children, and a \$2.8 million kickback scheme. New York: The New Press.

Fassin, D. (2014). "The ethical turn in anthropology: Promises and uncertainties." Hau: Journal of Ethnographic Theory 4 (1): 429-35.

Fassin, D. (2015). "Troubled waters: At the confluence of ethics and politics." In Four lectures on ethics: Anthropological perspectives, 175-210. Chicago: Hau Books.

Ferguson, J. (1999). Expectations of modernity: Myths and meanings of urban life on the Zambian copperbelt. Berkeley, CA: University of California Press.

Ferguson, J. (2015). Give a man a fish: Reflections on the new politics of distribution. Durham, NC: Duke University Press.

Fernández-Kelly, M. P. (1983). For we are sold, I and my people: Women and industry in Mexico's frontier. Albany, NY: State University of New York Press.

Fischer, E. F. (2014). The good life: Aspiration, dignity, and the anthropology of wellbeing. Stanford: Stanford University Press.

Foucault, M. (1977). Discipline and punish: The birth of the prison. Translated by Alan Sheridan. New York: Vintage Books.

Foucault, M. (1980). The history of sexuality, volume I: An introduction. Translated by Robert Hurley. New York: Vintage Books.

Foucault, M. (2008). The birth of biopolitics: Lectures at the Collège de France, 1978-79. Translated by G. Burchell. Houndmills, UK: Palgrave Macmillan.

Geertz, C. (1973). The interpretation of cultures. New York: Basic Books.

Giddens, A. (1971). Capitalism and modern social theory: An analysis of the writings of Marx, Durkheim and Max Weber. Cambridge: Cambridge University Press.

Ginsburg, F. D., L. Abu-Lughod, \& B. Larkin, Eds. (2002). Media worlds: Anthropology on new terrain. Berkeley, CA: University of California Press.

Gorski, P. S., Ed. (2013). Bourdieu and historical analysis. Durham, NC: Duke University Press.

Graeber, D. (2009). Direct action: An ethnography. Oakland, CA: AK Press.

Graeber, D. (2015). "Radical alterity is just another way of saying 'reality': A reply to Eduardo Viveiros de Castro." Hau: Journal of 
Ethnographic Theory 5 (2): 1-41.

Greenhouse, C. J., Ed. (2010). Ethnographies of neoliberalism. Philadelphia, PA: University of Pennsylvania Press.

Gregory, S. (2007). The devil behind the mirror: Globalization and politics in the Dominican Republic. Berkeley, CA: University of California Press.

Gupta, A. (2012). Red tape: Bureaucracy, structural violence, and poverty in India. Durham, NC: Duke University Press.

Gusterson, H. \& C. Besteman, Eds. (2010). The insecure American: How we got here and what we should do about it. Berkeley, CA: University of California Press.

Hale, C. R. (2006). "Activist research v. cultural critique: Indigenous land rights and the contradictions of politically engaged anthropology." Cultural Anthropology 21 (1): 96-120.

Hale, C. R., Ed. (2008). Engaging contradictions: Theory, politics, and methods of activist scholarship. Berkeley, CA: University of California Press.

Hardt, M. \& A. Negri. (2000). Empire. Cambridge, MA: Harvard University Press.

Hart, K., J. L. Laville \& A. D. Cattani, Eds. (2010). The human economy. Cambridge: Polity Press.

Harvey, D. (2005). A brief history of neoliberalism. New York and Oxford: Oxford University Press.

Ho, K. (2000). Liquidated: An ethnography of Wall Street. Durham, NC: Duke University Press.

Hymes, D., Ed. (1972). Reinventing anthropology. New York: Pantheon Books.

Jameson, F. (1984). "Postmodernism, or the cultural logic of late capitalism." New Left Review 146 (July-August): 53-92.

Juris, J. S. (2008). Networking futures: The movements against corporate globalization. Durham, NC: Duke University Press.

Juris, J. S. (2012). "Reflections on \#Occupy Everywhere: Social media, public space, and emerging logics of aggregation." American Ethnologist 39 (2): 259-79.

Juris, J. S. \& A. Khasnabish, Eds. (2013). Insurgent encounters: Transnational activism, ethnography, and the political. Durham, NC: Duke University Press.

Kelly, T. (2013). "A life less miserable?" Hau: Journal of Ethnographic Theory 3 (1): 213-16.

Klein, N. (2007). The shock doctrine: The rise of disaster capitalism. New York: Henry Holt and Company.

Kurik, B. (s/d). "Emerging subjectivities in protest." Forthcoming in The Sage handbook of resistance, edited by S. Vallas and D. Courpasson. Thousand Oaks, CA: Sage Publications.

Lambek, M. (2010a). "Introduction." In Ordinary ethics: Anthropology, language, and action, edited by Michael Lambek, 1-36. New York: Fordham University Press.
Lambek, M., Ed. (2010b). Ordinary ethics: Anthropology, language, and action. New York: Fordham University Press.

Lancaster, R. (2010). "Republic of fear: The rise of punitive governance in America." In The insecure American: How we got here and what we should do about it, edited by $\mathrm{H}$. Gusterson and C. Besteman, 63-76. Berkeley, CA: University of California Press.

Low, S. (2010). "A nation of gated communities." In In The insecure American: How we got here and what we should do about it, edited by Hugh Gusterson and Catherine Besteman, 27-44. Berkeley, CA: University of California Press.

Lyotard, J. F. (1984). The postmodern condition. Translated by G. Bennington and B. Massumi. Minneapolis, MN: University of Minnesota Press.

Mamdani, M. (1996). Citizen and subject: Contemporary Africa and the legacy of late colonialism. Princeton, NJ: Princeton University Press.

Mankekar, P. (2015). Unsettling India: Affect, temporality, transnationality. Durham, NC: Duke University Press.

Martin, L. H., H. Gutman \& P. H. Hutton, Eds. (1988). Technologies of the self: A seminar with Michel Foucault. Amherst, MA: The University of Massachusetts Press.

Mazzarella, W. (2009). "Affect: What is it good for?" In Enchantments of modernity: Empire, nation, globalization, edited by Saurabh Dube, 291-309. London: Routledge.

Merry, S. E. (2000). Colonizing Hawai'i: The cultural power of law. Princeton, NJ: Princeton University Press.

Mizushima, H. (2007). Net Café Refugees and Poverty in Japan (film in Japanese). Tokyo: Nihon terebi hoso kabushiki kaisha.

Moore, M. (director). (1989). Roger and Me (film). Produced by Michael Moore.

Moore, M. (2009). Capitalism: A Love Story (film). Produced by Anne Moore, Michael Moore. Newman, Katherine S. 1993. Declining fortunes: The withering of the American dream. New York: Basic Books.

Ong, A. (1987). Spirits of resistance and Capitalist discipline: Factory women in Malaysia. Albany, NY: State Univerity of New York Press.

Ong, A. (1999). Flexible citizenship: The cultural logics of transnationality. Durham, NC: Duke University Press.

Ong, A. (2006). Neoliberalism as exception: Mutations in citizenship and sovereignty. Durham, NC: Duke University Press.

Ong, A. and S. J. Collier, Eds. (2005). Global assemblages: Technology, politics and ethics as anthropological problems. Malden, MA: Blackwell Publishing.

Ortner, S. B. (1984). "Theory in anthropology since the sixties." Comparative Studies in Society and History 26 (1):126-66.

Ortner, S. B. (1995). "Resistance and the problem of ethnographic refusal." Comparative Studies in Society and History 37 (1): 173-93. 
Ortner, S. B. (1998). "Generation X: Anthropology in a mediasaturated world." Cultural Anthropology 13 (3): 414-40.

Ortner, S. B. (2006). New Jersey dreaming: Capital, culture, and the Class of '58. Durham, NC: Duke University Press.

Ortner, S. B. (2011). "On neoliberalism." Anthropology of this Century, Issue 1, May. http://aotcpress. com/articles/ neoliberalism/.

Ortner, S. B. (2013a). Not Hollywood: Independent film at the twilight of the American Dream. Durham, NC: Duke University Press.

Ortner, S. B. (2013b). "Bourdieu and 'history.'" Review of Bourdieu and Historical Analysis, edited by Philip S. Gorski. Anthropology of this Century, Issue 8, October. http://aotcpress.com/articles/ bourdieu-history/.

Piketty, T. (2014). Capital in the twenty-first century. Translated by Arthur Goldhammer. Cambridge, MA: Belknap Press of Harvard University Press.

Rabinowitz, D. (2014). "Resistance and the city." History and Anthropology 25 (4): 472-87.

Razsa, M. \& A. Kurnik. (2012). "The Occupy Movement in Zizek's hometown: Direct democracy and the politics of becoming." American Ethnologist 39 (2): 238-58.

Reiter, R. R., Ed. (1975). Toward an anthropology of women. New York: Monthly Review Press.

Robbins, J. (2013). "Beyond the suffering subject: Toward an anthropology of the good." Journal of the Royal Anthropological Institute 19 (3): 447-62.

Rosaldo, M. Z. \& L. Lamphere, Eds. (1974). Woman, culture and society. Stanford: Stanford University Press.

Rose, N. (1996). "Governing 'advanced' liberal democracies." Foucault and political reason, edited by Andrew Barry, Thomas Osborne, and Nikolas Rose, 37-64. Chicago: University of Chicago Press.

Rutherford, D. (2016). "Affect theory and the empirical." Annual Review of Anthropology 45: (Volume publication date November 2016).

Sahlins, M. (1981). Historical metaphors and mythical realities: Structure in the early history of the Sandwich Islands Kingdom. Ann Arbor, MI: University of Michigan Press.

Said, E. W. (1978). Orientalism. New York: Vintage Books.

Salmond, A. (2014). "Transforming translations (part 2): Addressing ontological alterity." Hau: Journal of Ethnographic Theory 4 (1): 155-87.

Sanyal, K. (2007). Rethinking capitalist development: Primitive accumulation, governmentality, and post-colonial capitalism. London and New York: Routledge.
Scott, J. C. (1985). Weapons of the weak: Everyday forms of peasant resistance. New Haven, CT: Yale University Press.

Scott, J. C. (1990). Domination and the arts of resistance: Hidden transcripts. New Haven, CT: Yale University Press.

Sennett, R. (1998). The corrosion of character: The personal consequences of work in the new capitalism. New York: W.W. Norton \& Company.

Sklair, L. (1993). Assembling for development: The Maquila industry in Mexico and the United States. San Diego, CA: Center for U.S.-Mexico Studies.

Szwed, J. F. (1972). "An American anthropological dilemma: The politics of Afro-American culture." In Reinventing anthropology, edited by Dell Hymes, 153-81. New York: Vintage Books.

Theodossopoulos, D. (2014). "On de-pathologizing resistance." History and Anthropology 25 (4): 415-30.

Theodossopoulos, D. Ed. (2014). Rethinking resistance in the 21st century. History and Anthropology, Special Issue 25 (4).

Tsing, A. L. (2015). The mushroom at the end of the world: On the possibility of life in capitalist ruins. Princeton, NJ: Princeton University Press.

Urciuoli, B. (2010). "Neoliberal education." In Ethnographies of neoliberalism, edited by C. J. Greenhouse, 162-76. Philadelphia, PA: University of Pennsylvania Press.

Wacquant, L. (2009). Punishing the poor: The neoliberal government of social insecurity. Durham, NC: Duke University Press.

Walker, H. \& I. Kavedžija. (2015). "Introduction: Values of happiness." Hau: Journal of Ethnographic Theory 5 (3): 1-23.

Walley, C. J. (2013). Exit zero: Family and class in postindustrial Chicago. Chicago: University of Chicago Press.

Williams, B. (2010). "Body and soul: Profits from poverty." In In The insecure American: How we got here and what we should do about it, edited by H. Gusterson and C. Besteman, 224-37. Berkeley, CA: University of California Press.

Williams, R. (1977). Marxism and literature. New York and Oxford: Oxford University Press.

Wolf, E. (1982). Europe and the people without history. Berkeley, CA: University of California Press.

Wright, M. W. (2001). "The dialectics of still life: Murder, women, and Maquiladoras." In Millenial capitalism and the culture of neoliberalism, edited by Jean and John L. Comaroff, 125-46. Durham, NC: Duke University Press.

Yan, Y. (2011). "How far can we move away from Durkheim?: Reflections on the new anthropology of morality." AOTC Issue 2, October. 